Emerging, Experimental and Current Topics Relevantto Technology in Counselor Education, Supervision and Practice

Journal of Technology in Counselor Education and Supervision (ISSN 2692-4129)

Volume 1(1): 19 - 26

(C) 2021Journal of Technology in Counselor Education and Supervision https://doi.org/10.22371/tces/003

\title{
The Virtual Bug: Online Live Supervision of Telemental Health Counseling
}

\author{
Raul Machuca' \& Ashley Kums²
}

\begin{abstract}
In this article, the authors present a model for the provision of Online Live Supervision of Telemental Health Counseling (OLSTHC). The model includes its development context, logistical considerations, technology and communication needs, administrative requirements, as well as clinical recommendations. In addition, the authors address ethical, multicultural, as well as future research considerations in the implementation of OLSTHC. The authors developed this model as a response to the training disruptions brought about by the Covid-19 pandemic, and represents a feasible alternative for the implementation of a virtual counseling training clinic.
\end{abstract}

\section{Keywords}

live supervision, online supervision, telemental health counseling

The Online Live Supervision of Telemental Health Counseling (OLSTHC) model described here was born as a response to the changes imposed by the COVID-19 pandemic to our counseling training program, particularly our practicum and internship experiences. The purpose of this article is for the authors to present the OLSTHC model as a template for the implementation of counseling training, live supervision, and counseling services in a virtual environment. In this paper, the authors present the basic supervision-related concepts that are involved in the model. Based on their experience implementing the model, the authors describe next the model's development context, logistical considerations, technology and communication needs, administrative requirements, and clinical recommendations. Finally, some ethical, multicultural, as well as future research considerations in the implementation of OLSTHC are provided.

The incorporation of technology in live supervision has evolved from closed-circuit camera observations of adjacent rooms and communication with the supervisee via telephone, the use of a bug-in-the-ear system (Boylston \& Tuma, 1972), the incorporation of visual or text-based supervisor-supervisee communication or bug-in-the-eye (Klitzke \& Lombardo, 1991; Machuca

$1 \quad$ Barry University, Miami Shores, FL, USA

2 Barry University, Miami Shores, FL, USA et al., 2016; Miller et al., 2002; Neukrug, 1991; Scherl \& Haley, 2000; Smith et al., 1998), to a fully online live supervision and counseling experience that combines, voice and text communication as well as live interaction between the supervisor, the supervisee, and the client (Nadan et al., 2020). The model presented here represents the integration of online live supervision and telemental health counseling into a comprehensive learning experience facilitated by the use of technology.

\section{Supervision}

Clinical Supervision is an intervention provided by a senior member to a junior member of the same profession (Bernard \& Goodyear, 2019). Clinical supervision is an essential component of counselor training (Barnett et al., 2007) that serves to provide support, instruction, and professional guidance to supervisees with an emphasis on maintaining the welfare of clients, as well as to uphold the ethics of the counseling profession and the corresponding laws that govern its practice (Bernard \& Goodyear, 2019). The successful experience of clinical supervision is dependent upon a solid supervision relationship (Bernard \& Goodyear, 2019; Ghazali et al., 2018), the use

Corresponding Author: Raul Machuca, PhD, barry University, 11300 NE 2nd Avenue, Miami Shores, FL 33161-6695 email: rmachuca@barry.edu 
of effective methods and interventions for supervision (Bernard \& Goodyear, 2019; Bright \& Evans, 2019; Watkins et al., 2015), the formulation of clear and collaboratively developed goals (Bright \& Evans, 2019; De La Torre-Brooks, 2018), as well as appropriate supervision assessment practices (Bernard \& Goodyear, 2019; Crunk \& Barden, 2017). The supervision experience can be done both live and as a post-counseling session experience.

\section{Live Supervision}

Live supervision is defined as a method of supervision in which a clinical supervisor observes a counselorin-training during the counseling session in real-time, while directly intervening to provide guidance (Bernard \& Goodyear, 2019). It is a common supervision method employed in counseling training clinics and implies a live communication exchange between the supervisor and the supervisee during the allotted supervision time (Machuca, 2019; Machuca et al, 2016). A benefit of live supervision is the extended period of active engagement, which gives the supervisee different opportunities to interact with their supervisor through text, phone calls or computer screens. It also facilitates for supervisors to focus more directly on the implementation of the feedback given to supervisees (Moody et al., 2014). Live supervision can be done both in a traditional in-person format, and in a virtual or online format.

\section{Online Supervision}

Online supervision, also known as cyber supervision, is defined as the clinical supervision of counseling services done solely using virtual means (Bender \& Dykeman, 2016). During this exchange, the supervisor is not in the same physical location as the supervisee (Pennington et al., 2019). There are two forms of online supervision, asynchronous and synchronous.

\section{Asynchronous}

This type of online supervision involves the use of email and audio/video sharing that does not necessarily involve real-time communication (Bender \& Dykeman, 2016). It implies the review and discussion of information in a virtual environment using recorded events (Teo et al., 2015).

\section{Synchronous}

Also known as live supervision, this form of supervision involves the use of online chatting and audio/video sharing that occurs in real-time (Bender \& Dykeman, 2016). During synchronous online supervision, feedback is delivered immediately during and/or after session (Teo et al., 2015). Online synchronous supervision is possible through a stable internet connection, which requires the use of appropriate technology (Teo et al., 2015).

The advantages of online supervision include the possibility of working with supervisors regardless of their location (Pennington et al., 2019), greater flexibility in one's academic schedule (Crowell \& McCarragher, 2007), as well as the opportunity for mental health professionals to refine meta-skills such as critical thinking, in-depth self-reflection, effective problem solving, and empathetic understanding in a virtual environment (Crowell \& McCarragher, 2007). The combination of online and live supervision gives place to online live supervision.

\section{Online Live Supervision}

Online live supervision is defined as the virtual supervision of clinical counseling services provided in realtime (Pennington et al., 2019). Online live supervision uses audio and video technology, as well as effective chat devices for instant communication (Bender \& Dykeman, 2016). In online live supervision, the clinical supervisor watches a live counseling session via the internet and provides guidance to the supervisee simultaneously (Rousmaniere, 2014). When using this method of supervision, the supervisee and the client meet in person or through telehealth, while the supervisor is connecting to the live session through an online video conference platform. With the COVID-19 pandemic, there has been an emergence in the implementation of online live supervision to ensure the continuation of the counseling training experiences for programs that rely on live supervision, as well as to ensure that field experience requirements are met (Nadan et al., 2020).

Through online live supervision, counselors are given the opportunity of accessing different modalities of supervision; they also have a greater choice of who their supervisor can be (Vaccaro \& Lambie, 2007). It decreases travel expenses while allowing the supervisor to be synchronously present during the session (Crowell \& McCarragher, 2007). There is also an ease of technological communication through the use of online chatting and text messages (Nadan et al., 2020). Finally, the use of online live supervision eliminates the need for supervisees to provide recordings of their counseling sessions for supervision purposes. It also minimizes the risks associated with the transmission or transportation of such recordings.

\section{Telemental Health Counseling}

Telemental health counseling is defined as the use of HIPAA compliant video and audio material for mental health professionals to provide counseling remotely (Sanchez Gonzalez et al., 2019). The advantages of telemental health counseling include reduced costs, less traveling, and accommodation to work schedules (Robinson \& Reiter, 2016), and benefits comparable to in-person counseling (Sanchez Gonzalez et al., 2019). 


\section{Supervision of Telemental Health Counseling}

The supervision of telemental health counseling as an extension of the traditional clinical supervision experience can take multiple configurations. It can be conducted in person with the supervisor and the supervisee in the same room, while the supervisee provides telemental health counseling to a client. It can also be provided completely online with the supervisor, the supervisee and the client located remotely and connected through an online platform (Nadan et al., 2020). In addition, the supervision of telemental health counseling can be done both synchronously (live supervision) or asynchronously (post-session supervision). When done synchronously, the supervisor interacts live with the supervisee while the supervisee provides telemental health counseling to a client.

\section{A Model for the Provision of Online Live Supervi- sion of Telemental Health Counseling}

Although live supervision has been traditionally used in counseling supervision at many on-campus counseling training clinics, the abrupt changes brought about by the COVID-19 pandemic, prompted the rapid development of online live supervision for counseling training and supervision clinics including our own. For us, the basic idea guiding this process was the possibility of offering similar supervision conditions for telemental health counseling to the ones offered by traditional live supervision of counseling, and at the same time, attending to the health guidelines established nationwide. Similar to other on-campus counseling training clinics (Nadan et al., 2020), our training clinic employs live supervision for master's and doctoral counseling students in their practicum and internship experiences. Because of the COVID-19 guidelines, our on-campus clinic implemented a protocol for the provision of telemental health counseling services by master's and doctoral level counseling students under the online live supervision of doctoral students and faculty supervisors.

Preliminary surveys conducted with faculty supervisors and students showed the Online Live Supervision of Telemental Health Counseling (OLSTHC) model offers both significant advantages as well as some challenges to the supervision experience. The main advantages highlighted the health protection benefits of the model under the COVID-19 circumstances, the amount and variability of support offered to supervisees because of the use of multiple technological applications, the richness of the supervision experience given the accessibility to resources, and the quality of services provided to clients because of the faster and more direct intervention the supervisor had on the supervisees' in-session performance. The main challenges reported were related to connection issues provoked by unreliable internet services, as well as some ethical considerations related to the location of supervisees and clients.

\section{Logistical Considerations}

In the Online Live Supervision of Telemental Health Counseling (OLSTHC) model the supervisor, the supervisee, and the client are connected simultaneously through a virtual telecommunication system, and the supervisee provides telemental health counseling services to a client located remotely. A supervisor who is also connected remotely is monitoring the counseling session live. The implementation of the OLSTHC method requires the use of a virtual platform where the counselor is able to offer telemental health counseling services to a client, and the supervisor can provide live supervision to the supervisee.

\section{The Process of Online Live Supervision of Telemental Health Counseling}

The online live supervision in our virtual counseling training clinic is provided to master's level counseling students by both faculty supervisors and doctoral student supervisors. Typically, our supervision groups consist of a faculty supervisor, a doctoral student supervisor completing their supervision internship requirements, and six master's level students in either their practicum or internship experience. The doctoral student supervisors provide online live supervision and the same time receive live supervision of their supervision by a faculty supervisor. On a typical day, the counselors-in-training and supervisors meet as a group 30 minutes before the first counseling sessions. During this time, the counselorsin-training discuss their plan for the upcoming sessions and receive any necessary feedback from the supervisors. These 30-minute online group supervision sessions continue after every telemental health counseling session to process each session and to prepare for the upcoming ones. After the initial 30-minute online group supervision session, the counselors-in-training, the supervisors, and the members of the reflecting team meet with the clients online. The reflecting team consists of all supervisors and supervisees, and its role is to observe the telemental health counseling sessions, and to provide clinical feedback and consultation.

During the OLSTHC experience the client, counselor in training and supervisor join the same telemental health counseling session. Although the supervisor may be visible during the initial counseling process with a client, the supervisor will observe the telemental health counseling session as anonymously as possible. As the supervisee works with the client, the supervisor provides live feedback to the supervisee on their performance using a secure communication application integrated into the telemental health platform, or through a separate and private communication application. 


\section{Technology and Communication Needs}

The implementation of the OLSTHC method requires the use of both online connectivity and communication enabling equipment such as computers, tablets, and mobile devices, as well as telemental health platforms and applications.

\section{Telemental Health Counseling Platforms}

A videoconference platform that allows more than two people to connect at the same time is a basic requirement for OLSTHC. Ideally, the counseling training setting can use an established telehealth platform as long as it allows for synchronous online observation of the telemental health counseling session by a supervisor. In our program, given the fact that we use both live supervision and reflecting teams, we opted for the use of a secure video conference system such as WebEx or Zoom because they allow simultaneous access. When selecting a telemental health platform we suggest the use of one that allows for secure and easy voice and video communication, the use of security features such as attendee verification and waiting rooms, the use of anonymity features such as name change, noise removal of entry/exit, as well as the removal of attendee lists from view. More detailed ethical considerations are described under the corresponding section below.

\section{Technological and Communication Apps}

The implementation of the OLSTHC method requires the use of videoconference and telemental health apps as described above, texting and communication apps, as well as electronic health record apps.

Texting and Communication Apps. Examples of these apps are Tigerconnect (2014) and Qliq (2020). These HIPAA compliant communication apps facilitate the secure sharing of text, voice, graphics and documents between the supervisor and the supervisee. In the Online Live Supervision of Telemental Health Counseling (OLSTHC) model, a secure communication app is used between supervisor and supervisee for the provision of live feedback during the telemental health counseling session. The use of a separate communication app, instead of the text feature commonly integrated into most telemental health platforms, was done to increase security and privacy in the communication between the supervisor and the supervisee, and to reduce the risk of having text messages between the supervisor and the supervisee being broadcasted to the client.

Electronic Health Record Apps. The use of electronic health records applications is essential in online live supervision of telemental health counseling because of the documentation challenges imposed by a virtual counseling and supervision experience such as inaccessibility to paper records, or limited access to nonweb based applications. Most counseling training clinics use web-based electronic health record systems. In our model, the supervisees have remote access to an electronic health record application, to complete the necessary documentation for their telemental health counseling and supervision experiences. Examples of web-based electronic health record applications are Simple Practice (2021) and CareCloud (2021). Counselors in training access the electronic health record system to complete all necessary documentation for their clients. Supervisors access the system to monitor, review and approve the counselor in training's documentation of their work with clients. Most electronic health record systems now include access to a telehealth platform however, the possibility of online live supervision of the telemental health session may require additional planning and logistics.

\section{Administrative Requirements Scheduling}

The on-campus training clinic administrative personnel do scheduling of clients. The scheduling of telemental health counseling clients is done either through the videoconference platform used for the telemental health counseling sessions or through the telemental health platform used by the training clinic. The scheduled client session is done with a designated counselor in training as a provider and includes the client, the counselor in training and the supervisor. At the time of the appointment the client, the counselor in training, and the supervisor join the session.

\section{Client Orientation}

The client orientation includes among other things, a description of the telemental health counseling, supervision and observation process, a presentation of the intake and service documentation to be signed electronically by the client, technological requirements to access telemental health counseling, as well as any other pertinent information related to payment, safety, confidentiality, and privacy. Clients are informed of the technology requirements for telemental health such as internet connection, a computer, tablet or smartphone equipped with camera and microphone. Clients are informed also of the presence of a supervisor and a reflecting team online but not visible to them (unless necessary), similar to the way observation of their sessions is done in the clinic.

\section{Orientation, Training and Credentialing}

Given the novelty of the OLSTMC experience for most supervisees, it is essential to provide a comprehensive orientation that includes technological requirements, access and logistics of the online live supervision and counseling sessions, as well as the diverse methods of online live supervision used. Our orientation includes information related to the practicum and internship process, requirements and documentation, the clinic administrative 
and clinical policies, as well as practices and documentation requirements. A HIPAA training is also part of the orientation to the virtual clinic, as well as a review of the emergency policy for telemental health services. All faculty supervisors and counselors in training complete formal training in the provision of telemental health and a mock OLSTMC session is conducted with the students and supervisors.

\section{Recommendations for Online Live Super- vision of Telemental Health Counseling (OLSTHC)}

This section draws mainly from the experience of implementing the OLSTHC model, in the context of practicum and internship experiences, at our campus counseling training clinic. In this section, the authors describe recommendations for the adaptation of traditional methods of supervision for Online Live Supervision of Telemental Health Counseling (OLSTHC). It also contains a description of OLSTHC interventions, as well as additional supervision considerations and recommendations.

\section{Methods of Online Live Supervision of Telemental Health Counseling}

The methods of OLSTHC described here represent an adaptation of traditional methods of supervision to the OLSTHC context.

Co-Counseling Supervision. When using this method of OLSTHC the supervisor remains visible and unmuted during the telemental health counseling session. As such, the supervisor and the supervisee have the opportunity of providing telemental health counseling to the client as a tandem. This method of live supervision is ideal when the supervisor wants to model specific skills for the supervisee or when the online counselor-in-training is working with clients who may require additional support. Examples of such clients are clients who present with current suicidal ideation, clients in crisis, and clients who present to sessions as particularly vulnerable.

Bug-in-the-Ear and/or Phone-In. For this method of online live supervision of telemental health counseling the online counselor-in-training wears a communication device that allows for the supervisor to provide live verbal feedback to the supervisee during the course of a telemental health counseling session. A way of facilitating this process is for the counselor in training to listen to the conversation with the client through the computer speakers, while wearing earphones or a headset to listen to the verbal feedback from the supervisor. The distance verbal communication between the supervisor and the supervisee can be established through a secure closed communication or telephone application system. A significant advantage of this method of OLSTHC is the promptness with which feedback can be offered to the counselor-in-training, and it is less intrusive. A known disadvantage is that listening to two sources of verbal information can be challenging for the supervisee (Machuca et al., 2016), and the risk of receiving excessive feedback (Scherl \& Haley, 2000). The logistics for the use of bug-in-the-ear and phone-in methods of live supervision in OLSTHC are essentially the same, except for the fact that the bug-in-the-ear method is used permanently during the session, while the phone-in method is typically used sporadically during the session.

Bug-in-the-Eye (BITE). The bug-in-the-eye method involves the provision of supervision feedback to the supervisee through a visual display. In our experience, we use a slightly modified form of the bug-in-the-eye method known as eye-bug supervision (Machuca et al., 2016). When using this method, the supervisor provides feedback to the supervisee through a parallel application in the supervisee's computer, a tablet, or a smartphone. The feedback is usually presented to the supervisee as text but it can also involve images, links, documents, etc., to which the supervisor may want the supervisee to attend or to use during the live telemental health counseling session.

Log-in Supervision. The log-in supervision method of OLSTHC is the virtual equivalent of the walk-in-method of live supervision (Bernard \& Goodyear, 2019). The most common example in our practice occurs when the supervisor who is observing the telemental health counseling session turns on the microphone and camera and joins the supervisee and the client in the counseling interaction.

Log-out supervision. The log-out supervision method of OLSTHC is the virtual equivalent of the consultation break (Bernard \& Goodyear, 2019) or the walk-out method of live supervision. The virtual consultation break can take the form of an announced pause of the telemental health counseling session, which can be pre-arranged and or suggested by the supervisee or the supervisor. During the log-out, the supervisee can leave the telemental health counseling session and join a separate telemental health counseling session. The supervisee can also mute their camera and microphone and call the supervisor to discuss any necessary aspect of the telemental health counseling session.

\section{Interventions in Online Live Supervision of Telemental Health Counseling}

Each of the methods of OLSTHC described above involves unique live supervision interventions that Bernard and Goodyear (2019) described in their general and traditional format. We used the cognitive behavioral model as our primary supervision model for the provision of OLSTHC. As such, most of the supervision interventions used correspond to this model of supervision such as the use of a supervision structure, agenda, and plans of action, as well as the facilitation of cognitive and behavioral 
interventions, use of didactic learning, and exploration of supervisee's affect, among others. Here, however, we will describe one supervision intervention that when used in the provision of OLSTHC is particularly unique, the provision of supervision feedback .

\section{Supervision Feedback}

The most direct form of OLSTHC intervention is the provision of instant and ongoing feedback to the supervisee in the course of a telemental health counseling session. As described before, supervision feedback is provided to the counselor-in-training via text primarily, and using the private chat option of the telemental health platform, or a separate HIPAA compliant application. In our case, we prefer the use of a separate communication application, TigerConnect (2014). It is helpful for the supervisor and the supervisee to agree on a common code for the presentation of the feedback, given its variability during an online live supervision session. Examples of codes that can be used are quotation marks for directives; e.g., "reflect meaning," parentheses for conceptualization; e.g., (the client seems to be experiencing the tyranny of the shoulds) or unmarked remarks for themes to keep present or questions to ask. As such, the type of feedback offered to the supervisee during the OLSTHC sessions varies depending on the client and supervisee's needs. The most common types of feedback provided include the following:

Supervisor Directives. This type of feedback corresponds to specific commands or directions given to the supervisee during the online live counseling session. Examples of these commands are "Reflect," "Explore the meaning of that," "validate," "normalize," etc.

Conceptualization Comments. These types of comments serve as the supervisor's contribution to the collaborative conceptualization process that occurs during an OLSTMC session. They express the supervisor and reflecting team (if presented) interpretation of the client's experience from a clinical perspective and according to a particular theoretical approach. An example of these types of comments is (The client presents distorted beliefs in every component of the cognitive triad).

Process Comments. Process comments include general process observations on the supervisee's interaction with the client, as well as performance feedback provided to the supervisee during the OLSTHC session. General process observations provide the supervisee with a thirdparty view of what is happening in the counseling session. An example of a general process observation is "it seems you experienced a minor impasse in the rapport building process today." Performance feedback refers to statements that provide an evaluative perspective of the supervisee's performance during the OLSTMC session. As such, they can express immediate approval or corrective feedback for the supervisee. In our experience, we prefer the use of short statements such as good job, great; as well as emojis such as a thumbs up or a happy face.

Information. This type of feedback refers to the provision of specific information that supports the work of the supervisee during the OLSTMC session. Examples of this type of feedback include demographic information and statistics, information about the prevalence of a condition, multicultural information, and medication information.

Response Modeling. This is the most diverse area of feedback provided by a supervisor during an OLSTMC session. When using this type of feedback, the supervisor's intent is for the supervisee to become an extension of themselves and stick to a more closely guided supervision intervention. The use of response modeling can correspond to situations when the client may benefit from more advanced counseling techniques or interventions. Examples of these types of feedback are specific statements and questions the supervisor wants the supervisee to use.

\section{Other Considerations and Recommendations}

It is recommended to offer supervisees the freedom to incorporate the feedback given. An important conversation during the supervisee orientation to the OLSTHC experience is the idea that, although there may be an expectation for the feedback given to be incorporated during the session, it is up to the supervisee to decide the most appropriate time to implement it.

It is also important for supervisors to provide a balanced amount of feedback. Less experienced supervisees may require feedback that is more frequent during the live supervision session. At the same time, supervisors need to attend to the supervisees' professional development and their need for increased self-efficacy.

The level of feedback offered is also an important consideration. In our experience, when supervisors provide live supervision they tend to use and suggest more advanced interventions for the supervisee. To address this issue, any complex guided intervention to be used during an online live supervision session needs to be discussed and practiced with the supervisee in advance of the actual session with a client.

Finally, unless it is an urgent matter that needs to be addressed immediately, the provision of corrective performance feedback during the OLSOTMC session should be limited. Positive performance feedback should be preferred. The corrective feedback can be provided during the debriefing session that typically follows a live supervision session.

\section{Legal and Ethical Considerations}

In addition to the legal and ethical considerations related to the provision of traditional counseling, telemental health counseling, supervision, and online supervision, 
the provision of Online Live Supervision of Telemental Health Counseling (OLSTHC) also involves some unique ethical considerations. In general, the main ethical framework for the provision of OLSTHC is the code of ethics of the American Counseling Association, and particularly Section $\mathrm{H}$ on distance counseling, technology, and social media (ACA, 2014). Using the ACA code of ethics as a framework, some of the most significant ethical considerations in the provision of OLSTHC are related to informed consent and security, knowledge of legal considerations, and records and web maintenance (ACA, 2014). More specifically, we want to highlight the need for a more specific informed consent for the provision of telemental health counseling that in our case involves the online live supervision component; confidentiality and privacy issues in the use of this method such as those concerning children and adults in need of assistance with technology; training and supervision issues and professional credentialing issues, such as the need for telemental health certification when possible; as well as safe production, transmission, and maintenance of documentation and records, which in a virtual scenario can be facilitated by the use of web-based electronic record keeping systems.

\section{Multicultural Considerations}

Some of the main multicultural issues we encounter during the experience of OLSTHC are related to age, primary language, and language accessibility, as well as socioeconomic status. In our experience, older adults may feel more intimidated about the use of technology, particularly when there is a sudden transition to it. Normalizing their experience, offering a more thorough orientation, and making the process for accessing services as simple as possible can be quite helpful.

In our training clinic, we have many clients, counselors, and faculty supervisors who speak multiple languages, including sign language. One multicultural issue we have encountered, however, is related to the use of interpretation services for faculty and doctoral supervisors, as well as members of the reflecting team who may not speak the language in which the client and the counselor-intraining communicate. When the counselor and the client speak different languages, the best option is to use the language interpretation feature some video conference platforms such as Zoom have. In terms of accessibility, the use of a group telemental health platform allows the presence of sign language interpreters, as well as automatic captioning of the dialogue for clients and or counselors with diverse hearing ability.

A final multicultural issue we often encountered was related to the client's and student's socioeconomic status. For clients, differences in financial situation or educational level need to be considered in the implementation of OLSTHC. Examples of specific issues we encounter were living conditions that made it difficult for the client to have a private place to connect at home. In some cases, the client had to connect from their car as this was the place they could be more in private. Access to technology and proficiency in the use of technology is another issue we encounter. Examples of this were clients who could only connect via their smartphones because they did not have access to a computer or internet. In the case of students, and perhaps because of the additional constraints imposed by the COVID-19 pandemic, there was a need to frequently remind them of the need to maintain confidentiality and privacy. There was also a need to support them in accessing the necessary technology they needed for their telemental health counseling and supervision experience. Being sensitive to the socio-economic realities of our supervisees and their clients is an important consideration in the provision of OLSTHC.

\section{Future Research}

The provision of Online live supervision of telemental Health Counseling (OLSTHC) in counseling programs and counseling training clinics has been catapulted these days by the physical distance imposed by the COVID-19 pandemic. The basic components of supervision, live supervision, online supervision and telemental health counseling have each been extensively researched. Yet, there is a significant need to research the confluence of these components with different populations, settings, and counseling experiences. From our experience, future research is needed on the impact of OLSTHC in the supervisee and client's experience, the supervision relationship in a completely virtual live context, the specific methods of OLSTHC, the online live supervision interventions for telemental health counseling, as well as the experience of supervisors and supervisors in training using OLSTHC. Finally, future research could also investigate general and specific components of the OLSTHC model for counseling training, practice and supervision.

\section{Conclusion}

Online Live Supervision of Telemental Health Counseling (OLSTHC) is a new and compounded supervision method. It is used by counselor education programs and counseling training facilities to respond to the needs of an evolving technological world (Nadan et al., 2020). Since supervisors, supervisees, and clients are no longer required to be in the same location or meet in a physical place, this method of supervision presents multiple advantages. The OLSTHC experience also presents an expansion in the logistic, administrative, clinical, legal, ethical, multicultural, and research-related considerations for the provision of clinical supervision.

\section{References}

American Counseling Association. (2014). 2014 ACA code of 
ethics. https://www.counseling.org/docs/default-source/ default-document-library/2014-code-of-ethics-finaladdress.pdf

Barnett, J. E., Erickson Cornish, J. A., Goodyear, R. K., \& Lichtenberg, J. W. (2007). Commentaries on the ethical and effective practice of clinical supervision. Professional Psychology: Research and Practice, 38(3), 268-275.

Bender, S., \& Dykeman, C. (2016). Supervisees' perceptions of effective supervision: A comparison of fully synchronous cybersupervision to traditional methods. Journal of Technology in Human Services, 34(4), 326-337.

Bernard, J. M., \& Goodyear, R. K. (2019). Fundamentals of clinical supervision (6th ed.). Pearson.

Boylston, W., \& Tuma, J. (1972). Training of mental health professionals through the use of the 'bug in the ear. The American Journal of Psychiatry, 129(1), 92-95.

Bright, S., \& Evans, A. M. (2019). Supervision development and working alliance: A survey of counseling supervisors. The Journal of Counselor Preparation and Supervision, 12(1). Retrieved from https://repository.wcsu.edu/ jcps/vol12/iss $1 / 1$

CareCloud, Inc. (2021). CareCloud (Version 0.2.87) [Mobile App]. https://www.carecloud.com

Crunk, A.E., \& Barden, S.M. (2017). The common factors discrimination model: an integrated approach to counselor supervision. The Professional Counselor, 7, 62-75.

De La Torre-Brooks, A, N. (2018). A qualitative examination of master's level counseling students' experiences of the supervisory working alliance and the use of online supervision. Online Theses and Dissertations. 562.

Ghazali, N. M., Jaafar, W. M. W., \& Anuara, A.. (2018). Supervision outcomes as predictor to the supervisory relationship and supervision contextual factors: study on the internship trainee counsellors. MATEC Web of Conferences. 150. 05073.

Klitzke, M. J., \& Lombardo, T. W. (1991). A "bug-in-the-eye" is better than a "bug-in the-ear": A teleprompter technique for on-line therapy skills training. Behavior Modification, 15(1), 113-117

Crowell, L. F., McCarragher, T. (2007). Delivering a social work MSW program through distance education: an innovative collaboration between two universities, USA. Social Work Education. 26:4, 376-388, DOI: 10.1080/02615470601081688

Machuca, R. (2019). Live play therapy supervision. In Dugan, E., Vaugh, K., \& Camelford, K. (Eds.). Developing and Sustaining Play therapy Clinics (pp. 224-246). IG Global.

Machuca, R., Johnson, T., \& Moro, R. (2016). Tablet-based live supervision: Eye-Bug Supervision. VISTAS 2016 Summer Ed.

Miller, K. L., Miller, S. M., \& Evans, W. J. (2002). Computerassisted live supervision in college counseling centers. Journal of College Counseling, 5, 187-192.
Moody, S., Kostohryz, K., \& Vereen, L. (2014). Authentically engaged learning through live supervision: A phenomenological study. Counselor Education \& Supervision, 53(1), 19-33. doi:10.1002/j.1556-6978.2014.00046.x.

Nadan, Y., Shachar, R., Leshem, T., Levenbach, D., Rozen, R., Salton, N., \& Cramer, S. (2020). Behind the (virtual) mirror: online live supervision in couple and family therapy. Family Process. 59. 10.1111/famp.12573.

Neukrug, E. S. (1991). Computer-assisted live supervision in counselor skills training. Counselor Education and Supervision, 31(2), 132-138.

Pennington, M., Patton, R., \& Katafiasz, H. (2019). Cybersupervision in psychotherapy. In $\mathrm{H}$. Weinberg \& A. Rolnick (Eds.), Theory and practice of online therapy (pp. 79-95). Routledge.

QliqSOFT. (2020). Qliq. (Version 2.8.7) [Mobile App]. https:// www.qliqsoft.com

Robinson, P. J., \& Reiter, J. T. (2016). Behavioral consultation and primary care: A guide to integrating services (2nd ed.). Springer International Publishing. https://doi. org/10.1007/978-3-319-13954-8

Rousmaniere, T. (2014). Using technology to enhance clinical supervision and training. In C. E. Watkins \& D. Milne (Eds.), Wiley-Blackwell international handbook of clinical supervision (pp. 204-237). Wiley Publishers.

Sanchez Gonzalez, M. L., McCord, C. E., Dopp, A. R., Tarlow, K. R., Dickey, N. J., McMaughan, D. K., \& Elliott, T. R. (2019). Telemental health training and delivery in primary care: A case report of interdisciplinary treatment. Journal of clinical psychology, 75(2), 260-270. https:// doi.org/10.1002/jclp.22719

Scherl, C., \& Harley, J. (2000). Computer monitor supervision: A clinical note. The American Journal of Family Therapy, 28(3), 275-282. doi:10.1080/01926180050081702

Simple Practice, LLC, (2021). Simple Practice (Version 9.3.0) [Mobile App]. https://apps.apple.com/us/app/ simplepractice-practice-management/id738207604

Smith, R. C., Mead, D. E., \& Kinsella, J. A. (1998). Direct supervision: adding computer assisted feedback and data capture to live supervision. Journal of Marital and Family Therapy, 24(1), 113-25.

Teo, Y.H., McNamara, S., Romeo, G. \& Gronn, D. (2015). Enhancing practicum supervision with asynchronous and synchronous technologies. Universal Journal of Educational Research, 3(5), 322-327.

TigerConnect (2014). TigerConnect (Version 4.6.98) [Mobile App]. https://tigerconnect.com

Vaccaro, N., \& Lambie, G. W. (2007). Computer-based counselor-in-training supervision: Ethical and practical implications for counselor educators and supervisors. Counselor Education and Supervision, 47(1), 46-57. 